incapacity must have been known to their teachers, provided the teachers themselves be not utterly incapable".

The Education Act of 1870 inaugurated the setting up of School Boards, and in his papers Tyndall has left a speech which he made just prior to the first elections to these Boards. He deals with some of the problems which are to be faced. Among these he notes, first, the problem of choosing teachers of the right quality. "The mischief done by incompetent teachers is beyond calculation". The next problem is that of text-books. "Almost every whipster imagines he can write a school book, whereas it requires the rarest faculty to write such a book." Here it may be noted that two of his own books, "On Heat" and "On Sound", were used in several public schools. He goes on to say, "The question of books will be one of the most important which the School Boards have to consider". Furthermore, the Boards will also have to determine the subjects to be taught. To the "three R's" he would add an elementary knowledge of some portion of physical science, in which he would include : the elementary facts and principles of chemistry; the nature of air and water and their functions in the human system; the facts and principles of combustion; the elementary facts of light, sound, heat, electricity and magnetism. "With the proper class-books the teacher would have no difficulty in making these things a source of unflagging interest to his boys."

In attempting to summarize the part played by the Royal Institution in the teaching of science during the nineteenth century, one may point out three stages by which the Institution's influence has developed. For nearly the whole of the first half of the century the emphasis, as we have seen, was on attempts at direct teaching. From one point of view, it may be considered that these attempts failed, and yet we may claim that not sufficient attention has been paid to them and that in many ways the Royal Institution pioneered interesting and important experiments of lasting value. The middle of the century saw the attempts to break down prejudice against science as a means of education and to enable it to encroach upon the almost universal classical tradition. Here again it is not unreasonable to say that the Royal Institution played an important part. Finally, it is hoped that sufficient has been mentioned of the influence of John Tyndall to show that, when Webster at the beginning of the century said that "the establishment of the Royal Institution ought to be considered as the commencement of a new era in the history of science in this country", he was a prophet indeed.

\title{
CONDUCTION MEASUREMENTS AT LOW TEMPERATURES
}

A ONE-DAY symposium on measurements of conduction at low temperatures was held by the Low Temperature Group of the Physical Society on April 11 at the Institute of Physics, and was attended by about fifty people. The committee of the Low Temperature Group, under the chairmanship of Dr. P. H. Sykes, arranged the symposium in a form similar to that of previous one-day discussions on the subjects of liquid helium and of low-temperature distillation, since it was felt that meetings of this nature make a valuable contribution towards the discussion and dissemination of recent ideas and results. Such symposia afford an opportunity, especially to younger research workers, of discussing their work informally and in much greater detail than would be possible in the more crowded and formal programme of a largescale conference. While the time for each paper was restricted to fifteen minutes, almost as much time was allowed for discussion, so that in the course of the day it was possible to consider some thirteen contributions on several aspects of conduction measurements at low temperatures. In view of the informality of the symposium, no detailed record of the proceedings will be published.

The morning session, under the chairmanship of Dr. K. Mendelssohn (Clarendon Laboratory, Oxford), opened with a group of three papers on electrical and thermal conduction in semi-conductors.

Measurements, over the temperature range $2^{\circ} \mathrm{K}$.$300^{\circ} \mathrm{K}$., of the electrical conductivity and Hall effect in $n$-type indium antimonide with electron concentrations ranging from $2 \times 10^{18}$ per $\mathrm{cm}^{3}$ down to $5 \times 10^{13}$ per $\mathrm{cm} .^{3}$, were described by $\mathrm{Dr}$. E. H. Putley (Royal Radar Establishment, Malvern). The ultimate purpose of the work was to estimate the purity of the parent material from the amount of electron scattering by ionized impurities at low temperatures. Because of the very small effective electron mass in indium antimonide, the electron mobilitios are very high, rising to maxima as great as $700,000 \mathrm{~cm} .{ }^{2} / \mathrm{V}$. sec. below $77^{\circ} \mathrm{K}$., and the electron gas becomes degenerate at much lower concentrations than in other semi-conductors so that, for example, the average relaxation times of electrons have to be obtained by integrating exactly over all energies, instead of assuming the usual formulæ for the cases of Fermi-Dirac statistics at high electron densities and of classical statistics. A theoretical calculation of the relaxation time, assuming additive inverse mean free paths for acoustical and impurity scattering, fitted the data at all temperatures, except those for impurity concentrations less than $10^{15}$ per $\mathrm{cm} .^{3}$ at temperatures below $50^{\circ} \mathrm{K}$., for which the calculated conductivity exceeded the observed value by a factor of two or three. It is not yet possible to say whether this indicates a defect in the theory of impurity scattering or the presence of some additional scattering. It was pointed out by Dr. Ziman that the conventional use of the Born approximation in the theory of impurity scattering is incorrect; however, a more rigorous phase-shift calculation increases the discrepancy between the observed and calculated conductivitios.

Dr. J. A. Carruthers (Clarendon Laboratory, Oxford) described measurements he had made with Dr. J. F. Cochran of the thermal conductivity of a series of $n$-type single crystals of germanium, to which Group 3 impurities had been added in concentrations ranging from $10^{14}$ per $\mathrm{cm}^{3}$ to $10^{19}$ per $\mathrm{cm}^{3}$. The work was carried out between $4 \cdot 2^{\circ} \mathrm{K}$. and $0.2^{\circ} \mathrm{K}$. as an extension to lower temperatures of previous results obtained above $2^{\circ} \mathrm{K}$. In all the 
specimens heat was conducted by the lattice, and although in one of the pure $n$-type specimens with $10^{13}$ impurities per $\mathrm{cm}^{3}$ phonons were scattered by the boundaries with 15 per cent specular reflexion, in all the other specimens phonons were scattered by boundaries and by an additional scattering mechanism. The phonon mean free path for this additional mechanism varied with temperature as $T^{0.8}$ in an impurity-band specimen, and varied as $T^{-1.5}$ in highly degenerate specimens, in rough agreement with a theory by Ziman on the scattering of phonons by a quasi-free-hole band with a low density of holes. For the purer specimens, the additional mean free path was approximately constant below $1^{\circ} \mathrm{K}$. at values not correlated with the amount of doping impurities.

The aim of a theoretical contribution by Mr. I. C. Pyle and Dr. J. M. Ziman (Cavendish Laboratory, Cambridge) read by the former, on the scattering of phonons by a pair of impurity centres, was to explain the $T^{0.8}$ dependence on temperature of the phonon mean free path in impurity-band germanium, described in the preceding paper. Using a model proposed by Mott for lightly doped semi-conductors, which presupposes some degree of electrical compensation, the mean free path for scattering of phonons by second-order electron transitions between neighbouring pairs of impurity sites was shown to have the required decrease with temperature, and to reach a minimum value near $1^{\circ} \mathrm{K}$., but the interaction was not sufficiently strong to explain the experimental results. First-order transitions, involving the complete absorption of a phonon, give a weaker interaction, but this would be increased by the fluctuations in the energies of the bound electronic states due to the random distribution of the impurities.

Electrical conductivity and galvanomagnetic effects in metals formed the subject of the last four contributions to the morning sessions.

Dr. R. W. Powell (National Physical Laboratory) described measurements, made in conjunction with Miss J. A. Nicholls and Mr. R. P. Tye, of the electrical and thermal conductivities to low temperatures of chromium, rhodium, iridium, platinum, rhenium, tungsten, cobalt, nickel, palladium, ruthenium, osmium, silver, and gold. Measurements on the specimens (which were all supplied by Johnson, Matthey and Co., Ltd., with the exception of the cobalt, which was prepared in the Metallurgy Division of the National Physical Laboratory) were also made to higher temperatures, and compared with results from the national laboratories in Canada and Australia. A study was made of the effects of heat treatment on the conducting properties of chromium in the form of a specially prepared electrodeposited tube, and it revealed the existence of an electrical resistance anomaly at about $40^{\circ} \mathrm{C}$. after annealing, and of deviations from Matthiessen's rule.

In the first of the three papers on galvanomagnetic effects, Dr. R. T. Webber (U.S. Office of Naval Research) discussed measurements of the longitudinal and transverse electrical magneto-resistance of copper of extremely high purity, in fields up to 100,000 oersteds at $293^{\circ}, 77^{\circ}$, and $4.2^{\circ} \mathrm{K}$. At $4 \cdot 2^{\circ} \mathrm{K}$. the longitudinal magneto-resistance showed a clear-cut saturation, and all the results, except those on transverse magneto-resistance below 50,000 oersteds at $4 \cdot 2^{\circ} \mathrm{K}$., could be fitted to simple two-parameter analytical forms. Since the specimens were annealed polycrystalline wires of diameter greater than ten times the electron mean free path, no size effects were observed, yet the changes in resistance were smaller than those observed by previous workers on specimens in the form of strips of similar size. Kohler's rule was obeyed at $4 \cdot 2^{\circ}$ and $293^{\circ} \mathrm{K}$., but not at $77^{\circ} \mathrm{K}$. A similar breakdown of Kohler's rule in rhodium at $77^{\circ} \mathrm{K}$., described by the next speaker, Dr. B. R. Coles (Imperial College of Science and Technology, London), is attributed to the presence at this temperature of comparable scattering by impurities and by lattice waves, each mechanism being assumed to have a relaxation time with a different lattice-symmetry and dependence on temperature, although, as pointed out by Dr. Ziman, this assumption, which is reasonable, has not been studied in detail. Rhodium also shows a reversal in sign of the Hall coefficient at high magnetic fields, as observed in other metals, and the Hall effect results can be fitted to a simple two-band model, provided one uses values of electron and hole densities which seem unrealistic in any other context. Recent work on the changes in the Hall coefficients of rhodium, palladium, and silver on alloying have shown that these are due, especially at liquid-hydrogen temperatures, not only to changes in the electronic structure, but also in part to alterations in the scattering mechanisms; and a careful interpretation of the results is necessary. In a short contribution on the theory of galvano-magnetism, Mr. F. GarcíaMoliner (Cavendish Laboratory, Cambridge) stressed that, since the expression for the magneto-resistance contains a multiplier which involves the integration of a geometrical factor over the Fermi surface, even slight deviations of the Fermi surface from sphericity can produce large increases in the magneto-resistance. In lithium, the Fermi surface derived by Jones and Zener (with approximately 5 per cent anisotropy) gives the experimental order of magnitude for the magneto-resistance, while surfaces calculated more recently lead to results in disagreement with experiment by two orders of magnitude. The anisotropy also increases the Hall coefficients for the alkali metals by up to 3 per cent and for the noble metals by a rather larger factor sufficient to explain Dr. Coles's results on Hall coefficients.

The afternoon session, under the chairmanship of Dr. F. E. Hoare (University of Leeds), opened with three papers dealing with superconductors. Dr. L. Mackinnon (University of Leeds) reviewed previous ultrasonic conduction measurements in normal and superconducting metals, and described work in cooperation with Mr. A. Myers on absorption of ultrasound in tantalum and mercury. Different absorptions in superconducting and normal tantalum were observed, but the specimen used was not pure enough to show a large effect. Although in mercury, where a very pure specimen is easier to obtain, the effect is much more noticeable, the attenuation of longitudinal waves in the normal metal shows a peculiar behaviour on application of a transverse field. Further experiments on this effect are in progress; an earlier specimen has shown a good, if fortuitous, correlation with recent theories on the energy gap.

Measurements on the thermal conductivity of superconducting lead at temperatures close to $1^{\circ} \mathrm{K}$., at which lattice conduction predominates, were described by Dr. H. Montgomery (Atomic Energy Research Establishment, Harwell). It was found that small percentages of alloying material cause only Rayleigh scattering of the phonons, but that plastic deformation greatly reduces the lattice conduction, and it appears that dislocations are distributed at random in annealed material, whereas in strained 
material they are assembled in mosaic walls. To overcome the effects of self-annealing at room temperature, measurements on lead alloys strained at $4^{\circ} \mathrm{K}$. and on a harder metal, niobium, strained at room temperature, were made by Mr. P. M. Rowell (Clarendon Laboratory, Oxford). The thermal resistivity due to initially straining a niobium specimen varies as the 0.8 th power of the strain, and as the niobium and lead alloy specimens are strained progressively, the extra thermal resistivity varies with temperature as $T^{-2 \cdot 0}$, as expected from the work of Klemens and of van Bueren on dislocations. Pure lead specimens do not show a similar behaviour.

The last three papers dealt with various aspects of scattering by impurities.

The electrical resistance of molybdenum and tungsten specimens irradiated at $77^{\circ} \mathrm{K}$. has been measured at helium temperatures by $\mathrm{Mr}$. M. W. Thompson (Atomic Energy Research Establishment, Harwell). Increases in resistance are found to recover after thermal annealing, and activation energies have been determined for the principal recovery processes; these occur at $-170^{\circ} \mathrm{C}$. and $+150^{\circ} \mathrm{C}$. in molybdenum, and at $-80^{\circ} \mathrm{C}$. and $+320^{\circ} \mathrm{C}$. in tungsten. It was suggested that recovery in the lower temperature-ranges is due to interstitial atoms being released from traps, whereas that at higher temperatures is due to migration of vacancies. A resistance minimum observed in molybdenum near $10^{\circ} \mathrm{K}$. became less pronounced after irradiation and recovered its original form only after annealing at $1,500^{\circ} \mathrm{C}$. Miss J. N. Lomer (Clarendon Laboratory, Oxford) described work carried out with Dr. H. M. Rosenberg on a method for determining dislocation densities in $\alpha$-brass by measurements of lattice thermal conductivity at liquid-helium temperatures. It was found that the dislocation densities introduced by strain are independent of the percentage of zinc alloyed, but depend only on whether the deformation is by single or multiple slip. The dislocation densities reach saturation at strains corresponding to the flattening-out of the stress-strain curves, and these saturation values increase with increasing zine content. The method is useful for fatigued materials, where the dislocation densities for cyclic stress are found to be the same as for the equivalent tensile stress.

Experiments to determine the thermal resistance due to isotopes in crystals of isotopically enriched lithium fluoride were deseribed by Dr. R. Berman (Clarendon Laboratory, Oxford). The scattering of single phonons can be calculated with reasonable certainty, but it is difficult to deduce the resultant thermal resistance, because the amount of mutual phonon interaction is uncertain. For low concentrations of either lithium- 6 or lithium- 7 the resistance is two to three times less than for the limit of very strong mutual phonon interactions; it is hoped to measure crystals with high concentrations of isotopes to determine the thermal resistance when mutual interactions are relatively weak. J. A. Carruthers

\section{NEWS and VIEWS}

National Physical Laboratory: Dr. J. A. Pople

Dr. John Anthony Pople has been appointed superintendent of the newly created Basic Physics Division at the National Physical Laboratory. Dr. Pople, who was born in 1925 , graduated from Trinity College, Cambridge, being a 'wrangler' in the Mathematical Tripos. After a short period with the Bristol Aeroplane Co., he returned to Cambridge as a research student under the late Sir John LennardJones. His work in this field led to the award of a Smith Prize in 1950 and election to a fellowship at Trinity College in 1951. Since 1954 he has been a lecturer in mathematics at Cambridge. This year he has been awarded the Marlow Medal of the Faraday Society. Dr. Pople has made theoretical contributions to a number of branches of molecular physics. Working with Lennard-Jones, he took part in the general development of the molecular orbital description of the electronic structure of molecules and has since been concerned with its application to specific problems. In addition, he has worked on the statistical mechanical theory of thermodynamic, dielectric and transport properties of liquids and gases. More recently, he has spent some time in the laboratories of the National Research Council of Canada at Ottawa, working on the interpretation of nuclear magnetic resonance spectra. The present physics programme of the National Physical Laboratory has its origin in the Laboratory's responsibility for measurement of physical constants and has a strong classical flavour; measurement of high pressures, ultrasonics, thermal conductivity and strength of materials are typical items. New fields to which consideration is being given are nuclear magnetics resonance, high polymer physics, and other aspects of molecular physics, including biophysies.

\section{Chemistry at Khartoum :}

Prof. J. Miller

DR. JOSEPH MILLER, who is at present reader in physical organic chemistry in the University of Western Australia, has been appointed to the chair of chemistry in the University of Khartoum. Prof. Miller is a graduate of University College, London, where he obtained first-class honours in chemistry in 1939. On the outbreak of war, he entered the armed services and obtained a commission in the Royal Engineers. He served throughout the War in chemical warfare units and in various theatres in the Mediterranean, and was discharged in 1946 with the rank of captain. He returned to University College and completed his doctorate in 1948 with a thesis dealing with the hydrolysis of oxime esters. He then joined the staff of the University of Western Australia, where he built up an active research school in physical organic chemistry, the work of the group being directed mainly to the investigation of nucleophilic substitution in aromatic compounds. During 1954, he visited the United States as a Fulbright Visiting Lecturer and spent extensive periods at both the University of California and the University of South Carolina as well as visiting a number of other centres. Prof. Miller, who is married and has three children, proposes to take up his duties at Khartoum at the beginning of the academic year this month. 